\title{
Un modèle viscoélastique pour la réponse des mousses polymériques à la compression cyclique
}

\author{
Gianpietro Del Piero ${ }^{1, a}$ et Giampiero Pampolini ${ }^{1,2}$ \\ 1 Dipartimento di Ingegneria, Università di Ferrara, Via Saragat 1, 44100 Ferrara, Italie \\ 2 Laboratoire de Mécanique et d'Acoustique, 31 chemin Joseph-Aiguier, 13402 Marseille, France
}

Reçu le 20 avril 2009

\begin{abstract}
Résumé - Sous compression cyclique, les mousses polymériques montrent une perte progressive de résistance lorsque le nombre de cycles augmente. Le recouvrement de résistance après une période de repos suggère que ce comportement puisse être décrit par un effet de mémoire à long terme. On propose un modèle viscoélastique fondé sur la coexistence d'une énergie élastique non convexe et d'une dissipation de type visqueux. Des essais expérimentaux montrent la bonne correspondance entre le modèle et le comportement observé.
\end{abstract}

Mots clés : Mousse polymérique / localisation des déformations / énergie non convexe / dissipation visqueuse / mémoire à long terme

\begin{abstract}
A visco-elastic model for polymeric foams under cyclic compression. A block of polyurethane foam subject to cyclic compression exhibits a softening phenomenon as the number of cycles increases. In particular, one observes a stepping down of the loading curves while the unloading curves remain the same. In tests performed by the authors, it has been observed a partial recovery of the initial stiffness after a resting period was observed. This behaviour can be described as a long memory effect. We propose a viscoelastic model, coupling a non convex energy with a dissipation of viscous type. The model is in a good qualitative agreement with the experiments.
\end{abstract}

Key words: Polymeric foams / strain localization / nonconvex energies / viscous dissipation / long-term memory

\section{Introduction}

Au cours d'essais de chargement cycliques en compression uniaxiale, conduits sur des cylindres de mousse de polyuréthane, on peut observer deux phénomènes intéressants : une localisation des déformations en bandes perpendiculaires à la direction de compression, et une perte progressive de résistance à la charge augmentant avec le nombre de cycles.

Le phénomène de localisation des déformations est bien connu et de nombreux auteurs ont proposé des modèles pour l'expliquer $[1,4,5,9]$. Le plus souvent, ces modèles se basent sur une approximation où les parois bordant la cellule sont représentés par des poutres et la localisation des déformations est expliquée par des instabilités de flambage. Par contre, dans le modèle proposé par les auteurs en [15], les échantillons sont modélisés comme un milieu continu élastique et homogène avec une énergie de déformation à deux puits, et l'instabilité est interprétée

a Auteur pour correspondance : dlpgpt@unife.it comme un changement progressif de phase, selon le schéma bien connu développé par exemple dans [16]. Avec une identification convenable des paramètres constitutifs du modèle, on a obtenu un bon accord entre la simulation et les résultats expérimentaux.

Toutefois ce modèle purement élastique n'est pas capable de décrire complètement la réponse des mousses polymériques lorsqu'elles sont soumises à une compression cyclique.

En effet, il ne peut pas décrire :

1. la dépendance de la réponse à la vitesse de chargement ;

2. la perte progressive de résistance à la charge augmentant avec le nombre de cycles;

3. l'effet de mémoire, qui consiste en un recouvrement de la résistance initiale après une phase de repos.

La dépendance de la réponse à la vitesse de chargement est une caractéristique des matériaux visqueux.

Le deuxième phénomène est semblable à ce que l'on observe pour les élastomères chargés en traction, qui est 
(a)

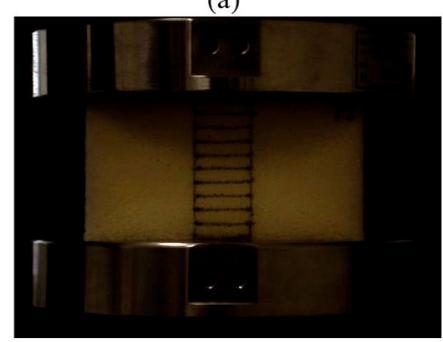

(d)

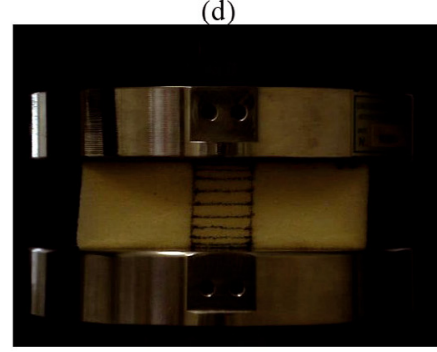

(b)

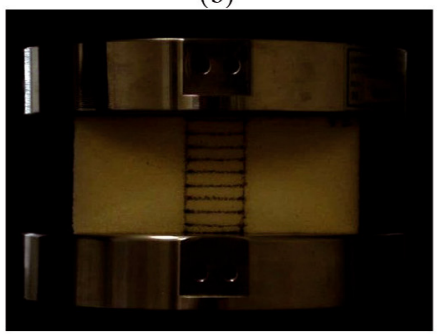

(e)

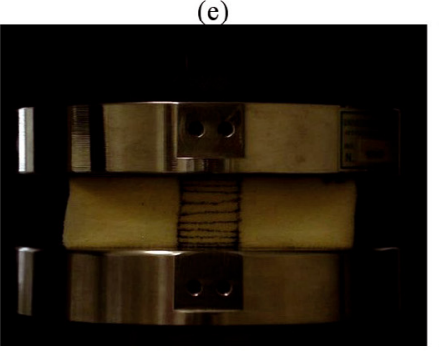

(c)
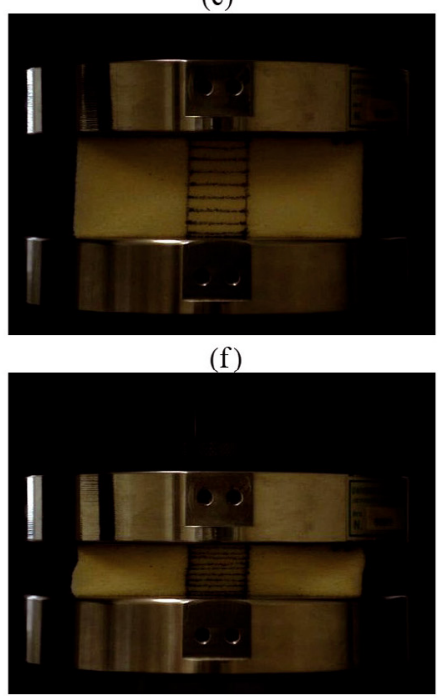

Fig. 1. Localisation des déformations.

appelé « effet Mullins » dans la littérature. Dans le modèle de Mullins et Tobin [14], la perte de résistance est attribuée à la rupture des liaisons entre la matrice polymérique et les particules de carbone. En se basant sur cette idée, beaucoup de modèles, qui décrivent macroscopiquement la rupture des liaisons avec une variable d'endommagement, ont été proposés [2,3,10,12].

Pour les mousses polymériques, le recouvrement de la résistance à la charge avec le temps suggère qu'un effet de mémoire à long terme soit apte à décrire le comportement cyclique.

Dans cette communication, on propose un modèle viscoélastique fondé sur la coexistence d'une énergie élastique non convexe et d'une dissipation de type visqueux. Les essais expérimentaux montrent une bonne correspondance entre le modèle et le comportement observé.

\section{Les essais expérimentaux}

On a effectué des essais en compression sur des échantillons (dimensions $100 \times 100 \times 50 \mathrm{~mm}$ ) de mousse de polyuréthane à cellules ouvertes, de qualité commerciale, en utilisant une machine d'essais INSTRON 4467, avec un capteur de force de $500 \mathrm{~N}$. On a posé une précharge de 2-3 N pour garantir un bon contact entre l'échantillon et le plateau supérieur de la machine d'essais. Tous les essais ont été effectués en déplacement contrôlé.

\subsection{Compression cyclique et localisation des déformations}

On a effectué des essais en compression cyclique, sur trois échantillons différents, en imposant une vitesse de la traverse mobile de $5 \mathrm{~mm} / \mathrm{min}$. À la charge, le sens du mouvement de la traverse est inversé lorsque l'on atteint un déplacement de $35 \mathrm{~mm}$ et, à la décharge, lorsque la force est nulle. L'essai est arrêté après sept cycles de charge et décharge. Les courbes moyennes forceélongation sont montrées sur la figure 2a. Sur cette figure et sur les suivantes, le terme «stress » indique la force divisée par l'aire initiale, et le terme « déformation » indique le rapport entre le déplacement de la traverse mobile et l'épaisseur de l'échantillon.

La courbe de réponse à la charge est composée de deux branches ascendantes divisées par un long plateau. Comme évoqué dans la littérature $[1,17]$, durant la phase de plateau, on observe une localisation des déformations en bandes perpendiculaires à la direction de la compression, cf. figure 1. Ce phénomène est dû à la présence de vides, qui provoquent l'écrasement progressif des cellules.

Lorsque le nombre de cycles augmente, on observe une perte progressive de résistance à la charge alors que celleci reste la même pendant les phases de décharge. Cette perte est importante en passant du premier au deuxième cycle, et elle devient faible après 4 ou 6 cycles [5].

Ce comportement ressemble au phénomène de « softening », connu comme effet Mullins, qui peut être observé pour les élastomères chargés lors d'essais en traction [13].

Pour comprendre l'influence des propriétés visqueuses sur ce comportement cyclique, on a effectué à nouveau les essais de compression cyclique sur les mêmes échantillons après une phase de repos de 33 jours. Les courbes de réponse sont montrées sur la figure $2 \mathrm{~b}$. On observe un recouvrement partiel de la résistance à la charge. Ceci confirme le bien fondé de la prise en compte de la viscosité.

\subsection{Essais de relaxation et essais en compression à différentes vitesses}

On a effectué des essais de relaxation de $72 \mathrm{~h}$ sur trois échantillons différents, en imposant un déplacement de $35 \mathrm{~mm}$ avec une vitesse de la traverse de $250 \mathrm{~mm} / \mathrm{min}$. 
(a)

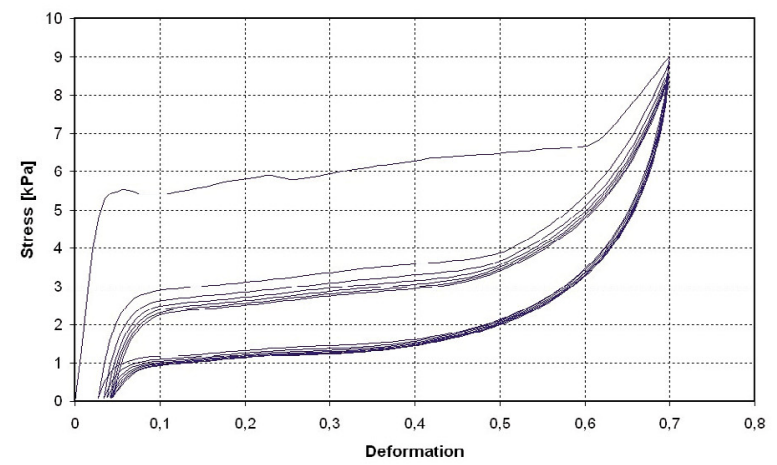

(b)

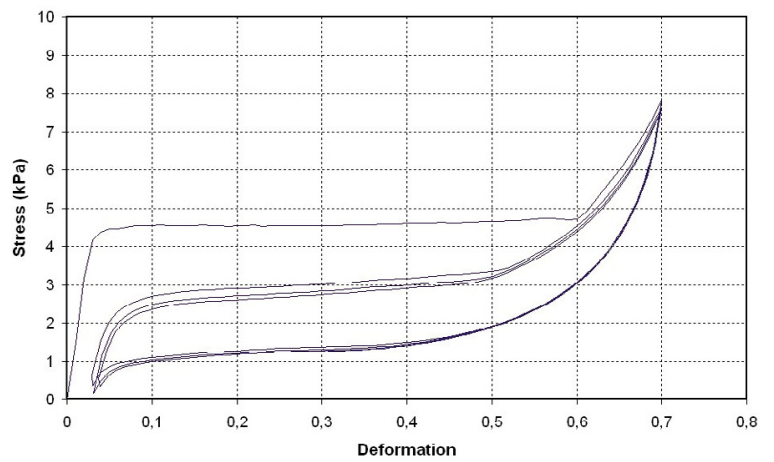

Fig. 2. Courbes expérimentales en compression cyclique sur échantillons vierges (a) et après une phase de repos de 33 jours (b).

(a)

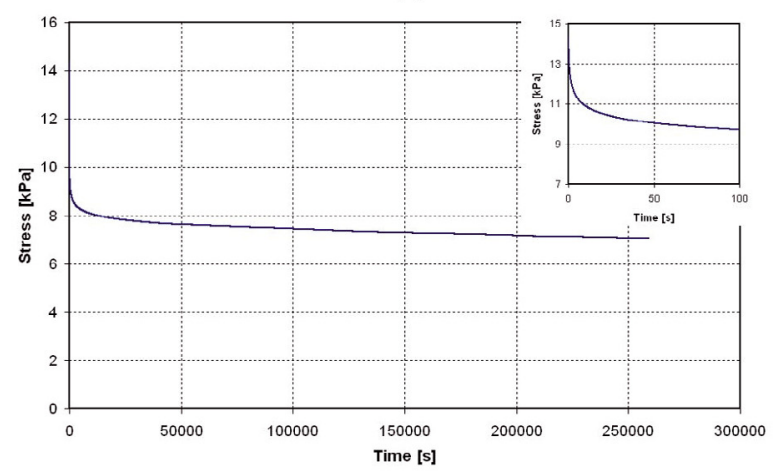

(b)

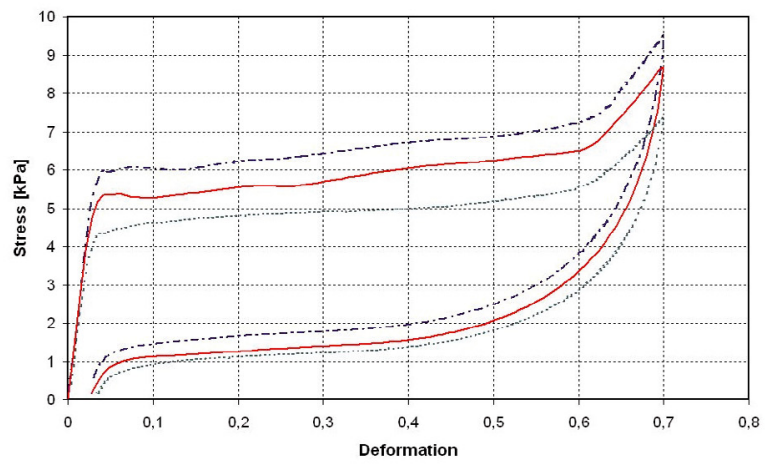

Fig. 3. (a) Courbe de relaxation. (b) Courbes force-élongation aux différentes vitesses, $0,1 \mathrm{~mm} / \mathrm{min}$ (ligne pointillée) $5 \mathrm{~mm} / \mathrm{min}$ (ligne pleine) et $100 \mathrm{~mm} / \mathrm{min}$ (ligne tiretée).

La force a été mesurée avec un intervalle d'échantillonnage d'une minute. La courbe moyenne est montrée sur la figure 3a. On observe une relaxation très rapide suivie d'une autre très lente, qui n'est pas encore terminée après trois jours.

On a effectué des essais en compression cyclique avec différentes vitesses de la traverse mobile, $0,1,5$, et $100 \mathrm{~mm} / \mathrm{min}$. Pour chaque vitesse, trois échantillons ont été testés; les courbes moyennes sont montrées sur la figure $3 \mathrm{~b}$. On observe une dépendance de la réponse à la vitesse de chargement. L'influence de la vitesse est plus importante à la charge, où on observe une translation vers le haut du palier.

\section{Le modèle rhéologique}

\subsection{La chaîne de ressorts}

Dans cette section, on décrit brièvement le modèle purement élastique, introduit en [15], où la mousse est représentée par une chaîne de ressorts, avec une énergie de déformation non convexe, et la localisation des déformations est décrite comme un changement de phase progressif.

On suppose que la mousse est composée de $N$ couches de cellules, on représente chaque couche par un ressort non linéaire (Figs. 4a et 4b). De plus, on suppose que tous les ressorts ont la même énergie de déformation non convexe $w$, figure $4 \mathrm{c}$. La courbe de réponse de chaque ressort est composée de deux branches ascendantes reliées par une branche descendante, figure $4 \mathrm{~d}$. On prend en compte le cas où l'élongation totale $N \varepsilon_{0}$ de la chaîne $\varepsilon_{0}$ est imposée. Cette condition au bord se traduit par la relation suivante :

$$
\sum_{i=1}^{N} \varepsilon_{i}=N \varepsilon_{0},
$$

où $\varepsilon_{i}$ désigne l'élongation du $i$-éme ressort.

L'énergie totale du système est la somme des énergies de déformation des ressorts. La condition d'équilibre, obtenue par l'annulation de la dérivée première de l'énergie totale, impose que la force de rappel de tous les ressorts soit la même. De plus, on peut montrer [16] que si l'on considère un nombre assez grand de ressorts, la condition de minimum de l'énergie totale impose que les élongations des ressorts soient dans l'une des deux branches ascendantes. Nous dirons que le ressort est en phase A si son élongation est dans la première branche ascendante et en phase B si celle-ci est dans la seconde branche ascendante. Toute configuration d'équilibre stable est caractérisée par $m$ ressorts en phase $\mathrm{A}$, et $n-m$ ressorts en phase $\mathrm{B}$, avec $m$ entre 0 et $n$. Pour comprendre la réponse de la chaîne de 


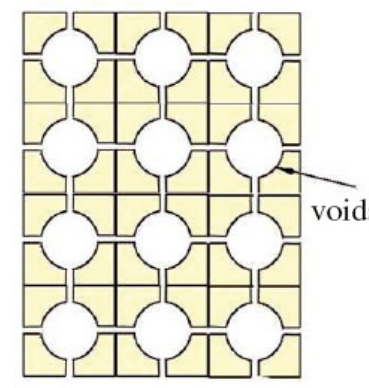

(a)

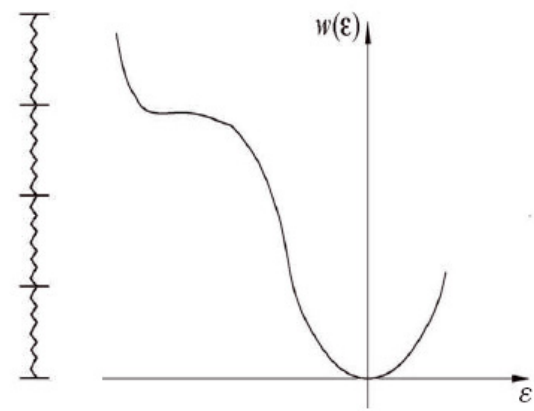

(c)

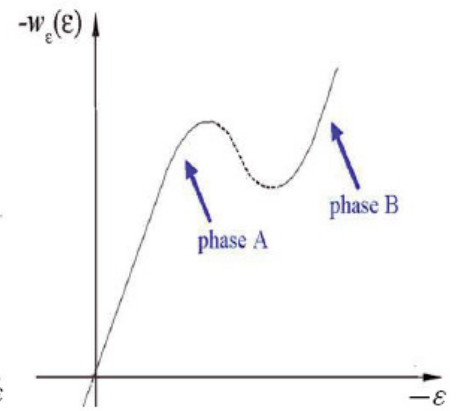

(d)

Fig. 4. Représentation de couches de cellules (a) comme une chaîne de ressorts (b) avec énergie de déformation non convexe (c). (d) Courbe de réponse de chaque ressort.

(a)

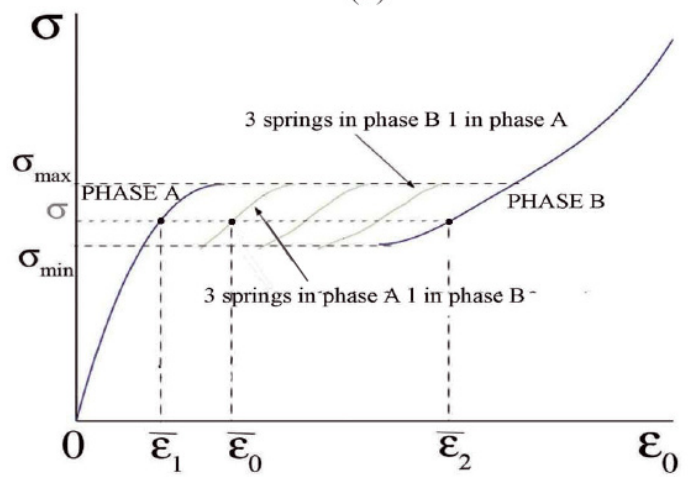

(b)

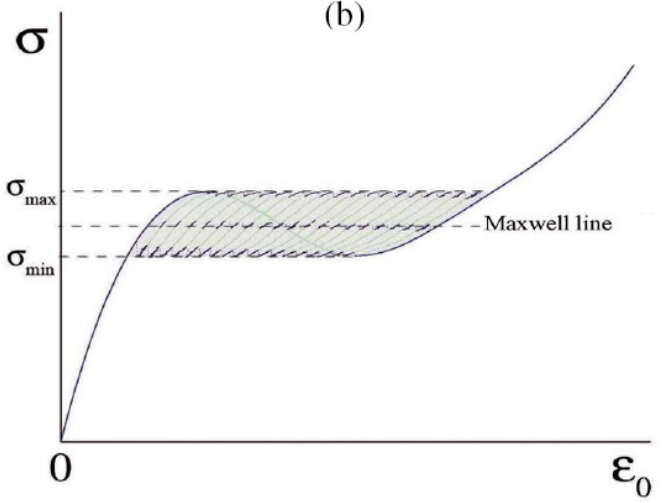

Fig. 5. Courbe de réponse d'une chaîne de 4 ressorts (a) et de 20 ressorts (b).

ressorts, on regarde la figure 5 où les chemins d'équilibre stable pour un système de quatre ressorts sont montrés. Lorsque le système est chargé à partir de la configuration initiale, la réponse suit la première branche ascendante jusqu'à ce que $\sigma$ atteigne la valeur $\sigma_{\max }$. En ce point, si l'on augmente l'élongation totale de la chaîne, le système saute à la branche d'équilibre stable la plus proche correspondant à la configuration avec un ressort en phase $\mathrm{B}$ et trois ressorts en phase $\mathrm{A}$. Le système suit cette branche jusqu'à son extrémité, puis il « saute » à la configuration avec deux ressorts en phase B. Donc on a un changement progressif de phase des ressorts. Lorsque tous les ressorts ont changé de phase, le système suit la seconde branche ascendante, qui correspond à la configuration avec tous les ressorts en phase B. À la décharge, le comportement est analogue mais la réponse oscille autour de la valeur $\sigma_{\text {min }}$. Si on augmente le nombre de ressorts, le nombre de branches bi-phase augmente, et l'amplitude des sauts décroît (Fig. 5b)

\subsection{L'amortisseur}

On considère un amortisseur avec une loi de comportement viscoélastique linéaire de type Boltzmann-Volterra,

$$
\sigma(t)=\int_{-\infty}^{t} G(t-s) \dot{\varepsilon}(s) \mathrm{d} s
$$

où $G$ est la fonction de relaxation, $t$ est le temps, et le point indique la dérivée temporelle. Si on prend en compte des histoires de chargement où $\varepsilon(s)=0$ pour $s \leq 0$, la réponse de l'amortisseur peut être écrite

$$
\sigma(t)=\int_{0}^{t} G(t-s) \dot{\varepsilon}(s) \mathrm{d} s
$$

On suppose que la fonction de relaxation est une loi puissance,

$$
G(t)=\frac{k}{\Gamma(1-\alpha)} t^{-\alpha}
$$

où $\Gamma$ est la fonction spéciale gamma, $k$ est une constante positive, et $0<\alpha \leq 1$.

Un amortisseur avec une loi de comportement (2) et une fonction de relaxation de type (4) correspond à l'élément gouverné par la loi à dérivées fractionnaires, que Koeller a appelé « spring-pot » [8],

$$
\sigma(t)=k \frac{\partial^{\alpha}}{\partial t^{\alpha}} \varepsilon(t)
$$



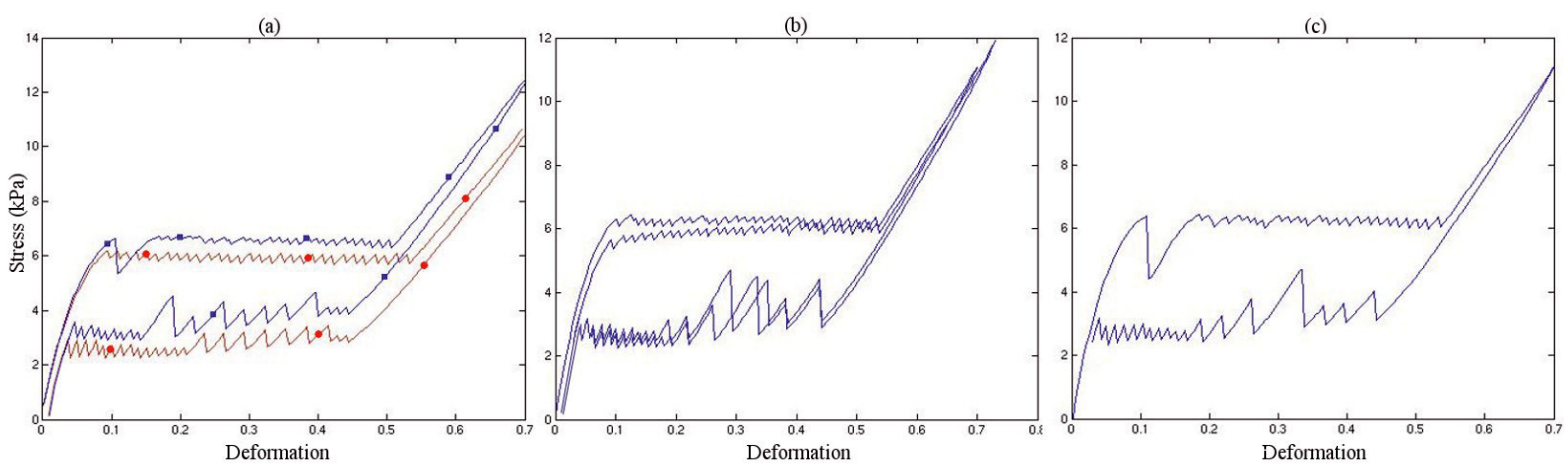

Fig. 6. (a) Courbes de réponse pour un système de 30 éléments : (a) deux essais à différentes vitesses de chargement 5 mm/min (marqueurs ronds) et $100 \mathrm{~mm} / \mathrm{min}$ (marqueurs carrés). (b) essai en compression cyclique sur échantillons vierges (b) et après une phase de repos de $72 \mathrm{~h} \mathrm{(c).}$

Si $\alpha=1$, la loi (5) se réduit à $\sigma=k \dot{\varepsilon}$. Pour les détails sur le calcul fractionnaire, on renvoie le lecteur à $[6,11]$. Le calcul fractionnaire a été largement utilisé pour décrire le comportement de matériaux polymériques, qui montre une dépendance non linéaire à la vitesse de déformation, et une relaxation très lente [7].

\subsection{Le modèle complet}

Nous présentons le modèle complet, obtenu en associant en parallèle à chaque ressort à énergie non convexe un amortisseur du type de celui décrit dans le paragraphe précédent. Si on appelle $\varepsilon_{i}$ l'élongation du $i$-ème élément et $\varepsilon_{0}$ l'élongation imposée, on a toujours la condition (1). De plus, la force transmise entre tous les éléments doit être la même. Cette force est la somme d'un terme élastique, dû aux ressorts, et d'un terme dissipatif dû aux amortisseurs,

$$
\sigma(t)=w_{\varepsilon}\left(\varepsilon_{i}\right)+\int_{0}^{t} G(t-s) \dot{\varepsilon}_{i}(s) \mathrm{d} s \quad i=1,2, \ldots, N
$$

Pour obtenir la réponse du système on doit adjoindre à la réponse des ressorts la contribution des amortisseurs. À l'équilibre, la réponse du système est celle de la chaîne de ressorts que l'on a décrite dans la section 3.2. On peut comprendre le comportement du système (du modèle complet) à l'aide de la figure 6 , où l'on montre le comportement d'un système de 30 éléments pour différentes conditions de chargement : essais à différentes vitesses de chargement, figure $6 \mathrm{a}$, essais en compression cyclique sur échantillons vierges, figure $6 \mathrm{~b}$, et après une phase de repos de $72 \mathrm{~h}$, figure $6 \mathrm{c}$. Les résultats montrent que le modèle proposé donne une bonne description qualitative des trois aspects du comportement des mousses polymériques décrits en introduction. En effet, le modèle montre

- une translation vers le haut de la courbe de réponse autant à la charge qu'à la décharge, si la vitesse de chargement augmente, figure $6 \mathrm{a}$;
- une translation vers le bas de la courbe à la charge du deuxième cycle, figure $6 \mathrm{~b}$;

- l'invariance de la courbe à la décharge par rapport au nombre de cycles, figure $6 \mathrm{~b}$;

- une déformation résiduelle après un essai de chargedécharge, figure $6 \mathrm{~b}$;

- un recouvrement partiel de la résistance après une phase de repos de $72 \mathrm{~h}$, figure $6 \mathrm{c}$.

\section{Références}

[1] S.G. Bardenhagen, A.D. Brydon, J.E. Guilkey, Insight into the physics of foam densification via numerical simulation. J. Mech. Phys. Solids 53 (2005) 597-617

[2] M.F. Beatty, S. Krishnaswamy, A theory of stresssoftening in incompressible isotropic materials. J. Mech. Phys. Solids 48 (2000) 1931-1965

[3] A. Dorfmann, R.W. Ogden, A pseudo-elastic model for loading, partial unloading and reloading of particlereinforced rubber, Int. J. Solids Struct. 40 (2003) 26992714

[4] L.J. Gibson, M.F. Ashby, Cellular Solids : Structure and Properties, Cambridge University Press, second edition, 1997

[5] L. Gong, S. Kyriakides, On the crushing stress of open cell foams, Journal of Applied Mechanics 73 (2007) 807814

[6] R. Gorenflo, F. Mainardi, Fractional calculus : integral and differential equations of fractional order, Fractals and fractional calculus in continuum mechanics, in : A. Carpinteri, F. Mainardi (éd.), CISM Courses and Lectures, 378, Springer, Vienna, 1997, pp. 223-276

[7] N. Heymans, J.C. Bauwens, Fractal Rheological Models and Fractional Differential Equations for Viscoelastic Behaviour, Rheologica Acta 33 (1994) 210-219

[8] R.C. Koeller, Applications of Fractional Calculus to the Theory of Viscoelasticity, Journal of Applied Mechanics 51 (1984) 299-307

[9] R. Lakes, P. Rosakis, A. Ruina, Microbuckling instability in elastomeric cellular solids, J. Mater. Sci. 28 (1993) $4667-4672$

[10] A. Lion, A constitutive model for carbon black filled rubber, experimental investigations and mathematical representations, Continuum Mech. Thermodyn. 8 (1996) 153169 
[11] A. Lion, On the thermodynamics of fractional damping elements, Continuum Mech. Thermodyn. 9 (1997) 83-96

[12] C. Miehe, J. Keck, Superimposed finite elasticviscoelastic-plastoelastic stress response with damage in filled rubbery polymers, experiments, modelling and algorithmic implementation, J. Mech. Phys. Solids 48 (2000) 323-365

[13] L. Mullins, Softening of rubber by deformation, Rubber Chem. Technol. 42 (1969) 339-362

[14] L. Mullins, N.R. Tobin, Theoretical model for the elastic behavior of filler-reinforced vulcanized rubbers, Rubber Chem. Technol. 30 (1957) 555-571
[15] G. Pampolini, G. Del Piero, Strain localization in opencell polyurethane foams : experiments and theoretical model, Journal of Mechanics of Materials and Structures 3 (2008) 969-981

[16] G. Puglisi, L. Truskinovsky, Mechanics of a discrete chain with bi-stable elements, J. Mech. Phys. Solids 48 (2000) $1-27$

[17] Y. Wang, A.M. Cuitinho, Full-field measurements of heterogeneous deformation patterns on polymeric foams using digital image correlation, Int. J. Solids Struct. 39 (2002) 3777-3796 